\title{
REAL-TIME DETECTION AND RAPID MULTIWAVELENGTH FOLLOW-UP OBSERVATIONS OF A HIGHLY SUBLUMINOUS TYPE II-P SUPERNOVA FROM THE PALOMAR TRANSIENT FACTORY SURVEY
}

\author{
Avishay Gal-Yam ${ }^{1}$, Mansi M. Kasliwal ${ }^{2}$, Iair Arcavi ${ }^{1}$, Yoav Green ${ }^{1}$, Ofer Yaron $^{1}$, Sagi Ben-Ami ${ }^{1}$, Dong Xu ${ }^{1}$, \\ Assaf Sterniberg ${ }^{1}$, Robert M. Quimby ${ }^{2}$, Shrinivas R. Kulkarni ${ }^{2}$, Eran O. OfeK ${ }^{2,18}$, Richard Walters ${ }^{2}$, \\ Peter E. Nugent ${ }^{3}$, Dovi Poznanski ${ }^{3}, 18$, Joshua S. Bloom ${ }^{4}$, S. Bradley Cenko ${ }^{4}$, Alexei V. FilipPenko ${ }^{4}$, Weidong Li $^{4}$, \\ Jeffrey M. Silverman ${ }^{4}$, Emma S. Walker ${ }^{5}$, Mark Sullivan ${ }^{6}$, K. Maguire $^{6}$, D. Andrew Howell ${ }^{7,8}$, \\ Paolo A. Mazzali ${ }^{5,9,10}$, Dale A. Frail ${ }^{11}$, David Bersier ${ }^{12}$, Phil A. James ${ }^{12}$, C. W. Akerlof ${ }^{13}$, Fang Yuan ${ }^{14}$, \\ Nicholas LaW ${ }^{15}$, DereK B. Fox ${ }^{16}$, and Neil Gehrels ${ }^{17}$ \\ ${ }^{1}$ Department of Particle Physics and Astrophysics, Faculty of Physics, The Weizmann Institute of Science, \\ Rehovot 76100, Israel; avishay.gal-yam@weizmann.ac.il \\ ${ }^{2}$ Cahill Center for Astrophysics, California Institute of Technology, Pasadena, CA 91125, USA \\ ${ }^{3}$ Computational Cosmology Center, Lawrence Berkeley National Laboratory, 1 Cyclotron Road, Berkeley, CA 94720, USA \\ ${ }^{4}$ Department of Astronomy, University of California, Berkeley, CA 94720-3411, USA \\ ${ }^{5}$ Scuola Normale Superiore, Piazza dei Cavalieri 7, 56126 Pisa, Italy \\ ${ }^{6}$ Department of Physics (Astrophysics), University of Oxford, Keble Road, Oxford OX1 3RH, UK \\ ${ }^{7}$ Las Cumbres Observatory Global Telescope Network, Goleta, CA 93117, USA \\ ${ }^{8}$ Department of Physics, University of California, Santa Barbara, CA 93106-9530, USA \\ ${ }^{9}$ INAF-Osservatorio Astronomico, vicolo dell Osservatorio 5, I-35122 Padova, Italy \\ ${ }^{10}$ Max-Planck Institut für Astrophysik, Karl-Schwarzschild-Str. 1, D-85748 Garching, Germany \\ ${ }^{11}$ National Radio Astronomy Observatory, P.O. Box O, Socorro, NM 87801, USA \\ ${ }^{12}$ Astrophysics Research Institute, Liverpool John Moores University, Twelve Quays House, Egerton Wharf, Birkenhead CH41 1LD, UK \\ ${ }^{13}$ Randall Laboratory of Physics, University of Michigan, 450 Church Street, Ann Arbor, MI 48109-1040, USA \\ ${ }^{14}$ Research School of Astronomy and Astrophysics, The Australian National University, Cotter Road, Weston Creek, ACT 2611, Australia \\ ${ }^{15}$ Dunlap Institute for Astronomy and Astrophysics, University of Toronto, 50 St. George Street, Toronto M5S 3H4, Ontario, Canada \\ ${ }^{16}$ Astronomy and Astrophysics, Eberly College of Science, The Pennsylvania State University, University Park, PA 16802, USA \\ ${ }^{17}$ NASA Goddard Space Flight Center, Greenbelt, MD 20771, USA \\ Received 2011 January 23; accepted 2011 May 15; published 2011 July 19
}

\begin{abstract}
The Palomar Transient Factory (PTF) is an optical wide-field variability survey carried out using a camera with a $7.8 \mathrm{deg}^{2}$ field of view mounted on the 48 inch Oschin Schmidt telescope at Palomar Observatory. One of the key goals of this survey is to conduct high-cadence monitoring of the sky in order to detect optical transient sources shortly after they occur. Here, we describe the real-time capabilities of the PTF and our related rapid multiwavelength follow-up programs, extending from the radio to the $\gamma$-ray bands. We present as a case study observations of the optical transient PTF10vdl (SN 2010id), revealed to be a very young core-collapse (Type II-P) supernova having a remarkably low luminosity. Our results demonstrate that the PTF now provides for optical transients the realtime discovery and rapid-response follow-up capabilities previously reserved only for high-energy transients like gamma-ray bursts.
\end{abstract}

Key words: supernovae: general - supernovae: individual (PTF10vdl)

Online-only material: color figures, machine-readable table

\section{INTRODUCTION}

The study of cosmic explosions, the energetic events marking the death of stars, is a unique subfield of astrophysics in the sense that its research subjects are variable sources on timescales much shorter than human lifetimes. Furthermore, many physical aspects of these events can only be revealed by observations conducted shortly after explosion. An extreme case is that of a gamma-ray burst (GRB; e.g., Piran 2004; Woosley \& Bloom 2006; Nakar 2007), where the most energetic radiation is emitted within a few seconds after the explosion. The study of GRBs has seen much progress following the launch of the Swift satellite, enabling rapid multiwavelength follow-up observations of GRBs.

Investigation of optical transients has thus far lagged behind in this respect. Rapidly detecting optical transients from among the multitude of constant (and variable) sources visible in the data stream from wide-field optical surveys has proved

\footnotetext{
${ }^{18}$ Einstein Fellow.
}

challenging for existing systems. Initial progress in securing very early-time observations of optical transients was made with relatively smaller surveys such as the Lick Observatory Supernova Search (LOSS) using the Katzman Automatic Imaging Telescope (KAIT; e.g., Ganeshalingam et al. 2010) and the Texas Supernova Survey using the ROTSE telescopes (e.g., Quimby et al. 2007), or due to chance coincidences such as supernovae ( $\mathrm{SNe}$ ) occurring in fields being monitored due to previous supernova (SN) explosions (e.g., Stritzinger et al. 2002; Soderberg et al. 2008).

One of the main science goals of the Palomar Transient Factory $\left(\mathrm{PTF}^{19}\right.$ ) wide-field variability survey (Rau et al. 2009; Law et al. 2009) is to remedy this situation and to facilitate realtime discovery and prompt monitoring of optical transients. Following recent upgrades to the computing infrastructure and software that now process all PTF data in real time and disseminate internal alerts about transients, and finalization of the operational aspects required to make use of these discoveries,

19 http://www.astro.caltech.edu/ptf/ 
the PTF is now a real-time optical variability experiment. Targetof-opportunity (ToO) programs are in place to conduct rapid multiwavelength follow-up observations of PTF transients.

Here, we describe the PTF operational setup enabling realtime discovery and rapid ToO observations of optical transients. We describe in some detail a specific example, the discovery and multiwavelength follow-up campaign of the optical transient PTF10vdl. Our observations of this remarkably subluminous Type II-P SN demonstrate the scientific potential of data collected shortly after explosion.

\section{PTF REAL-TIME DETECTION AND PROMPT MONITORING}

PTF images (currently $11 \times 8.4$ megapixels per exposure or $184 \mathrm{MB}$ per full array readout) are recorded on computers inside the 48 inch Schmidt telescope dome at Palomar Observatory and are then rapidly transmitted to the National Energy Research Scientific Computing Center (NERSC) at Lawrence Berkeley National Laboratory (LBNL). A series of software components is then run in sequence (P. E. Nugent et al. 2011, in preparation), including image detrending, astrometry, image subtraction with respect to deep references produced from co-additions of previous PTF data, and object detection in the difference images.

All of these data are loaded into a database upon which a series of quality cuts to reject artifacts is performed, followed by a rough classification into four groups by the Oarical software. These groups include candidate $\mathrm{SNe}$, sources in galactic nuclei, variable point sources, and moving objects. Within $\sim 40$ minutes from image acquisition, internal alerts are circulated, informing interested collaboration members of recent discoveries and classifications. This selection process will be described in more detail in a forthcoming publication (J. S. Bloom et al. 2011, in preparation).

Once the automated discovery pipeline runs to completion, several additional processes begin. First, follow-up observations for the most promising candidates (candidate $\mathrm{SNe}$ or sources near known nearby galaxies) are robotically triggered. Optical imaging is scheduled at the robotic 60 inch $(1.5 \mathrm{~m})$ telescope (P60; Cenko et al. 2006) at Palomar Observatory, while an infrared follow-up request is sent to PAIRITEL. ${ }^{20}$ Next, manual scanning of recent discoveries is conducted. Taking advantage of the $10 \mathrm{hr}$ time difference between Israel and California, a duty astronomer at the Weizmann Institute monitors recent discoveries from Palomar during Israeli daytime, and can manually trigger additional follow-up observations if desired (see below). At the end of each night, good candidates are sent to be screened by the citizen-science "supernova zoo" program (Smith et al. 2011), while custom database queries are run by collaboration members with specific focused interests (local universe transients, e.g., Kasliwal et al. 2011; Type Ia SNe, e.g., Cooke et al. 2011; SN shock breakouts, e.g., Ofek et al. 2010). Objects tagged by the SN zoo volunteers are also manually reviewed by the Weizmann duty astronomer. Upon discovery of an object deemed of special interest, follow-up programs are triggered.

Between 2011 January 1 and April 1, there were 70 nights during which the survey was running for at least part of each night. During those nights, there were 732 automated alerts for extragalactic transients (so about 10 per night, on average), of which 296 were considered likely SNe, 208 likely active galactic nuclei (AGNs, mostly from known sources), and 228

\footnotetext{
20 http://www.pairitel.org/
}

were nuclear events of undetermined nature. Weizmann Duty astronomers saved 172 events as high-priority SN candidates for follow-up; of those 97 (56\%) were spectroscopically followedup, leading to the discovery of $79 \mathrm{SNe}(46 \%$, of which 64 were of Type Ia). The events observed spectroscopically but not determined to be SNe were CVs or other variable stars (9), four galaxy spectra (likely resulting from target $\mathrm{SNe}$ fading away, errors in spectroscopic acquisition, or spurious detections), four spectra which were of too low signal-to-noise ratio $(\mathrm{S} / \mathrm{N})$ to identify the nature of the target, and one asteroid. During spring/ summer time the PTF discovery rates are substantially higher, due to a combination of better weather at Palomar and the survey strategy, however, the real-time procedures described here were only perfected during summer 2010, and we therefore provide statistics based on the above period.

Rapid follow-up programs designed to monitor PTF discoveries (mostly in ToO mode) are in place. A partial list of currently active programs includes access to the Wise Observatory $1 \mathrm{~m}$ and 18 inch telescopes, which, with the benefit of the $10 \mathrm{hr}$ time difference with respect to Palomar, can provide rapid "samenight" confirmation imaging (see below). Additional ToO photometry can be obtained by the Las Cumbres Observatory Global Telescope network (LCOGT), while continued optical monitoring of SNe is conducted using the $0.75 \mathrm{~m}$ KAIT (Filippenko et al. 2001) at Lick Observatory and at the Liverpool Telescope (LT) in La Palma. ToO spectroscopic programs, as well as many classically scheduled nights, provide access to the $10 \mathrm{~m} \mathrm{Keck}$ telescopes, the $5 \mathrm{~m}$ Hale telescope at Palomar Observatory, the $3 \mathrm{~m}$ Shane telescope at Lick Observatory, the $8.1 \mathrm{~m}$ Gemini-North and Gemini-South telescopes, the $8.2 \mathrm{~m}$ Very Large Telescope units at the European Southern Observatory (ESO) site on Paranal, the $9.2 \mathrm{~m}$ Hobby-Eberly Telescope (HET) at McDonald Observatory, the $3.5 \mathrm{~m}$ Telescopio Nazionale Galileo (TNG; service queue mode) in La Palma (Spain), and the University of Hawaii $2.2 \mathrm{~m}$ telescope on Mauna Kea. There are also radio follow-up programs with the Extended Very Large Array (EVLA ${ }^{21}$ ), the Allen Telescope Array (ATA), and the Combined Array for Research in Millimeter-wave Astronomy (CARMA) facility (e.g., Carpenter 2010; Corsi et al. 2011). U1traviolet (UV), X-ray, and $\gamma$-ray observations are obtained with the Swift mission. These resources provide a high probability that multiwavelength follow-up observations for an interesting PTF target can be obtained on short timescales (hours). We exemplify this capability in the next section using observations of PTF10vdl. Other recent examples include PTF10fqs (Kasliwal et al. 2011), PTF10hjz (D. A. Frail et al. 2011, in preparation), and PTF10iya (Cenko et al. 2011).

\section{OBSERVATIONS OF PTF10vdl}

\subsection{Detection Timeline}

PTF10vdl was detected at the 48 inch Oschin Schmidt telescope (P48) on 2010 September 15.24 (UT dates are used throughout) and was not detected in previous images obtained by the survey up to 3.9 days earlier. PTF10vdl is located at $\alpha=23^{\mathrm{h}} 05^{\mathrm{m}} 48^{\mathrm{s}} .88, \delta=+03^{\circ} 31^{\prime} 25^{\prime \prime} .5$ (J2000). The survey data were downlinked to the NERSC computing center at LBNL and were processed in real time. The object was discovered by the automated Darical software shortly (43 minutes) after detection, and an internal notification was broadcast. The

\footnotetext{
21 The Very Large Array is operated by the National Radio Astronomy Observatory, a facility of the National Science Foundation operated under cooperative agreement by Associated Universities, Inc.
} 

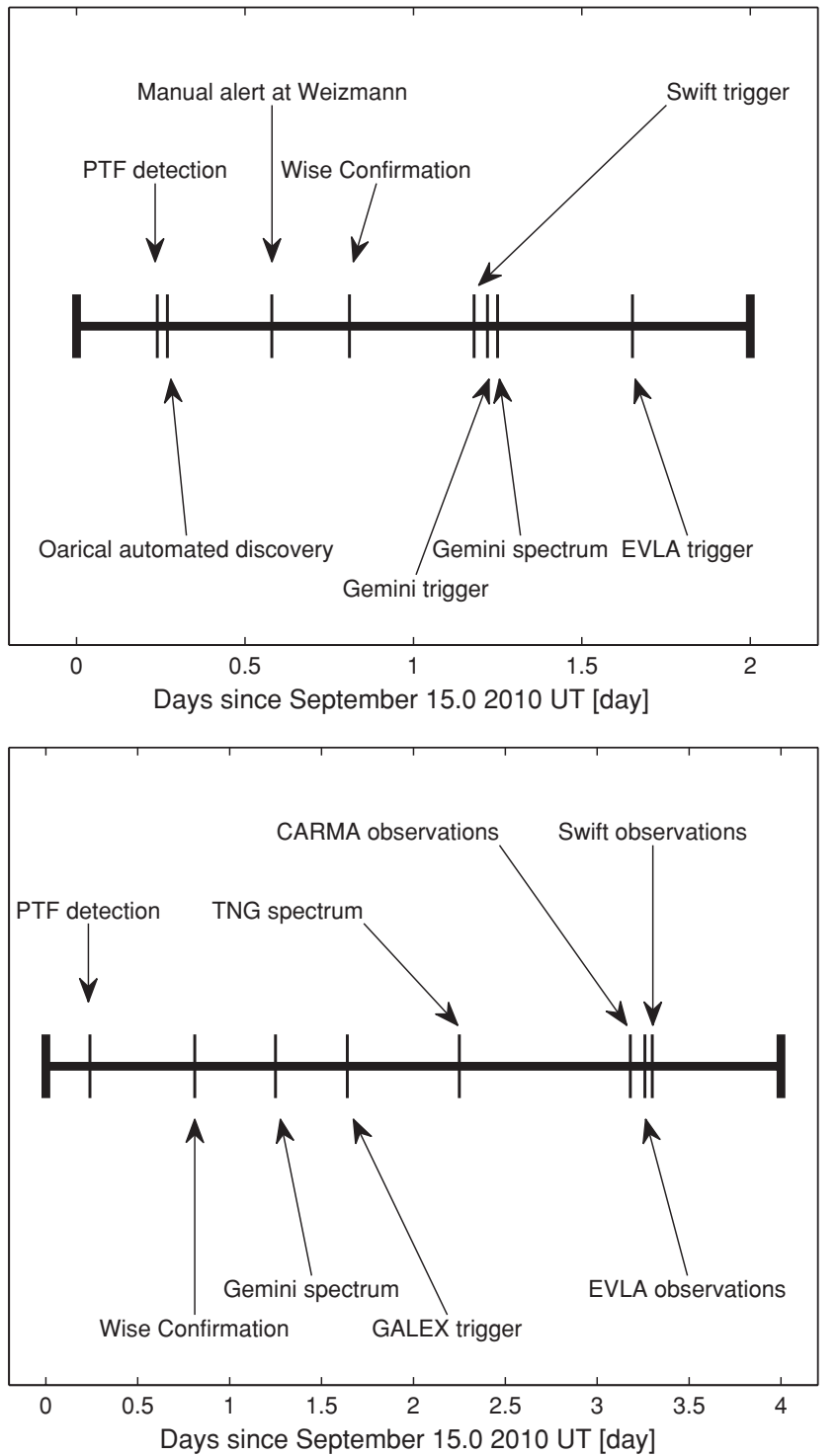

Figure 1. Top: time line of activities following the initial detection of PTF10vdl at Palomar. Bottom: multiwavelength observations of PTF10vdl; see the text for details.

Weizmann astronomer on duty (Y.G.) scanned the automated discovery reports as they came in during Israeli daytime and sent an alert concerning PTF10vdl and other recent discoveries of interest on September 15.58. The association of this optical transient with a nearby host galaxy (NGC 7483 at redshift $z=0.016475$; Springob et al. 2005, via NED) focused our attention on this event. The timeline of activities following that alert is presented in Figure 1 (top), and included obtaining a confirmation image of the object using the Wise Observatory $1 \mathrm{~m}$ telescope, followed by triggering approved PTF ToO programs using the Swift satellite, the EVLA radio telescope, and spectroscopy using the Gemini-North and TNG telescopes.

\subsection{Follow-up Observations}

After the rapid spectroscopic confirmation of PTF10vdl (Figure 2), we obtained multiwavelength observations of this object. Swift UV and X-ray observations were rapidly scheduled, but could not be carried out until September 18 (Kasliwal et al. 2010a) due to Sun-angle constraints (XRT cooling). No X-rays or $\gamma$-rays were detected; optical/UV observations were reduced
Table 1

PTF10vdl: Multicolor Photometry

\begin{tabular}{lcccc}
\hline \hline $\begin{array}{l}\text { Julian Date } \\
\text { (day) }\end{array}$ & $\begin{array}{c}\text { Magnitude } \\
(\mathrm{mag})\end{array}$ & $\begin{array}{c}\text { Error } \\
(\mathrm{mag})\end{array}$ & Band & Telescope \\
\hline 2455450.77 & 21.34 & 99.99 & $r$ & $\mathrm{P} 48$ \\
2455450.82 & 21.47 & 99.99 & $r$ & $\mathrm{P} 48$ \\
2455459.13 & 18.86 & 0.10 & $r$ & $\mathrm{P} 60$ \\
2455460.16 & 18.94 & 0.05 & $r$ & $\mathrm{P} 60$ \\
2455463.12 & 18.80 & 0.07 & $r$ & $\mathrm{P} 60$ \\
2455468.40 & 18.65 & 0.05 & $r$ & $\mathrm{P} 60$ \\
2455468.41 & 18.73 & 0.07 & $r$ & $\mathrm{P} 60$ \\
2455469.19 & 18.71 & 0.04 & $r$ & $\mathrm{P} 60$ \\
2455470.38 & 18.85 & 0.03 & $r$ & $\mathrm{P} 60$ \\
2455473.33 & 18.81 & 0.03 & $r$ & $\mathrm{P} 60$ \\
\hline
\end{tabular}

Notes. Multiband photometry of PTF10vdl. All optical ground-based data are calibrated onto the SDSS grid. Swift data are calibrated following Poole et al. (2008). The P60, LT, and Wise gri data calibrate consistently onto the SDSS grid, while small constant offsets had to be applied to the LCOGT, P48, and KAIT data; values reported here include these offsets. Values of 99.99 in the error column indicate that the values in the magnitude column are upper limits.

(This table is available in its entirety in a machine-readable form in the online journal. A portion is shown here for guidance regarding its form and content.)

with custom scripts based on the prescription of Poole et al. (2008) and are shown in Figure 4 and Table 1. GALEX UV spectroscopy was triggered, but could not be carried out due to the proximity of PTF10vdl to the UV-bright star $\beta$ Psc.

CARMA and EVLA radio observations were obtained on September 18 as well, yielding upper limits (Carpenter et al. 2010; Kasliwal et al. 2010b; Figure 1, bottom). The EVLA observations were made on September 18.26 at a center frequency of $8.46 \mathrm{GHz}$; we did not detect radio emission from the position of PTF10vdl down to a $3 \sigma$ limit of $105 \mu \mathrm{Jy}$. The mean frequency of the CARMA observations was $97.5 \mathrm{GHz}$ with a total bandwidth of $8 \mathrm{GHz}$. PTF10vdl was not detected, with a measured flux density of $0.13 \pm 0.13 \mathrm{mJy}$.

Rapid ToO spectroscopic observations with Gemini-North were conducted with the Gemini-N Multi-Object Spectrograph (GMOS; Hook et al. 2004), configured with a $1^{\prime \prime}$ slit and the R400 and B600 grisms (720 s and 520 s, exposures, respectively), yielding one of the earliest spectra obtained of any SN (Figure 2). Shortly thereafter we obtained service queue-mode spectroscopy with the $3.5 \mathrm{~m}$ TNG using the Device Optimized for the Low Resolution (DOLORES) spectrograph, configured with the LR-B grism and a 1" slit. Two exposures of $1200 \mathrm{~s}$ each were taken at the parallactic angle (Filippenko 1982) under good conditions with 1"4 seeing. Both spectra were reduced within IRAF in a standard manner. In both spectra, emission lines of $\mathrm{H}$ and $\mathrm{He}$ are superposed on a very blue continuum, indicating that this was a young Type II SN. The remarkably strong, highexcitation $\mathrm{He}$ II lines are indicative of the initially high temperatures. Additional spectroscopic monitoring was conducted with the $9.2 \mathrm{~m}$ HET, 3 m Lick, $5 \mathrm{~m}$ Palomar, and $10 \mathrm{~m}$ Keck telescopes (Figure 3). Photometric monitoring was carried out using the $1 \mathrm{~m}$ Wise, $2 \mathrm{~m} \mathrm{LT}$, P60, KAIT, and $2 \mathrm{~m}$ LCOGT Faulkes-North telescopes. The resulting light curve (Figure 4 and Table 1) shows that PTF10vdl is a remarkably underluminous SN II-P.

Digital copies of our data can be downloaded directly from the Weizmann Institute of Science Experimental Astrophysics Spectroscopy System ${ }^{22}$ (O. Yaron et al. 2011, in preparation).

\footnotetext{
22 http://www.weizmann.ac.il/astrophysics/wiseass/
} 


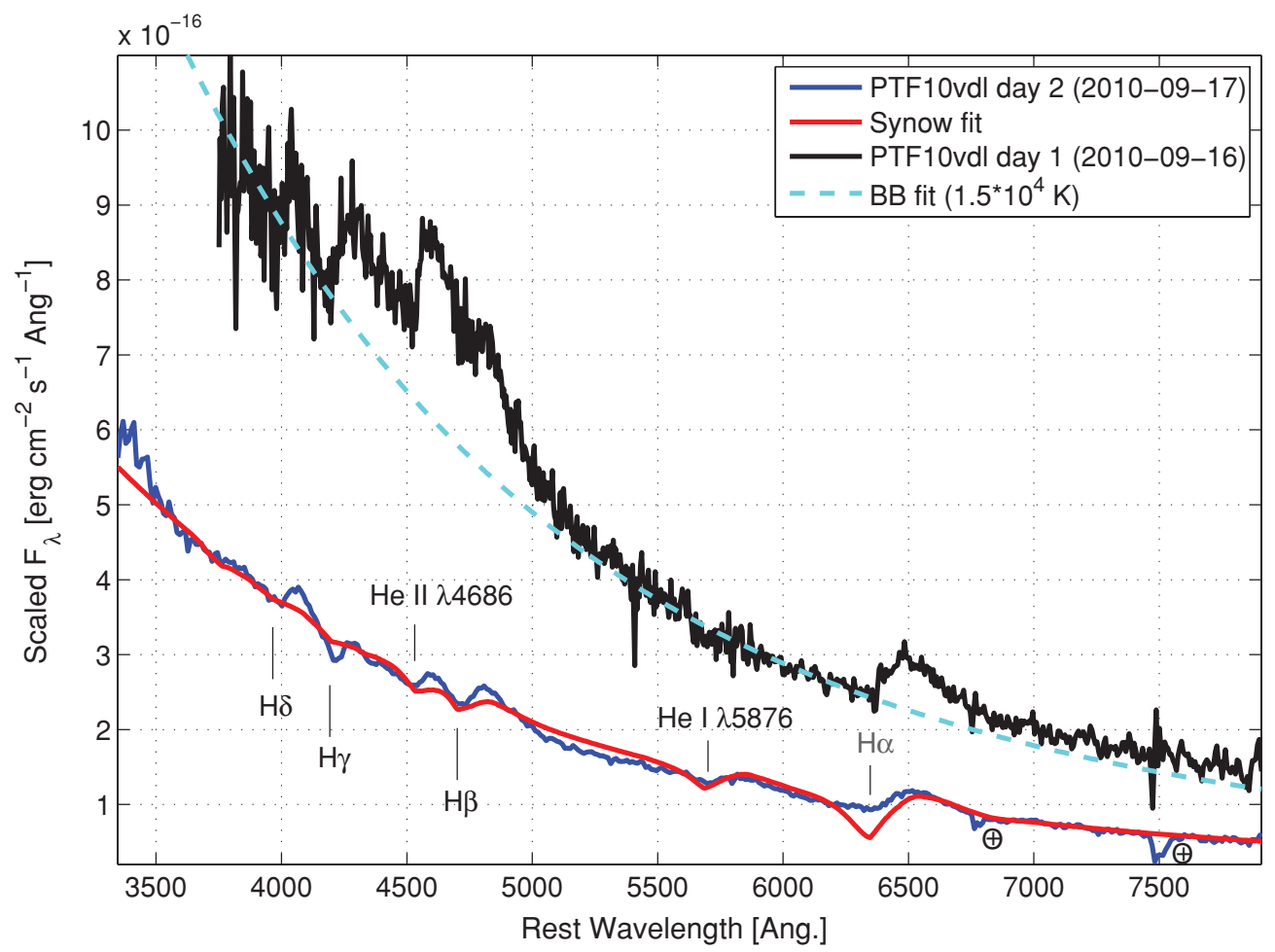

Figure 2. Early-time spectra of PTF10vdl. The Gemini spectrum (top) exhibits a very blue continuum (a blackbody curve with $T_{\mathrm{BB}}=1.5 \times 10^{4} \mathrm{~K}$ is shown for reference; cyan dashed curve), with superposed line emission dominated by hydrogen and helium. A higher signal-to-noise ratio spectrum of PTF10vdl obtained with the $3.5 \mathrm{~m}$ TNG about 1 day after the initial Gemini spectrum was fit using the SYNOW parametric spectral synthesis code; line identifications are marked (using the same $T_{\mathrm{BB}}$ as above). Note the strong He II emission, suggesting a very hot photosphere. The spectra indicate that this is a very young SN II, with very few similar spectra on record (e.g., for SN 2006bp; Quimby et al. 2007).

(A color version of this figure is available in the online journal.)

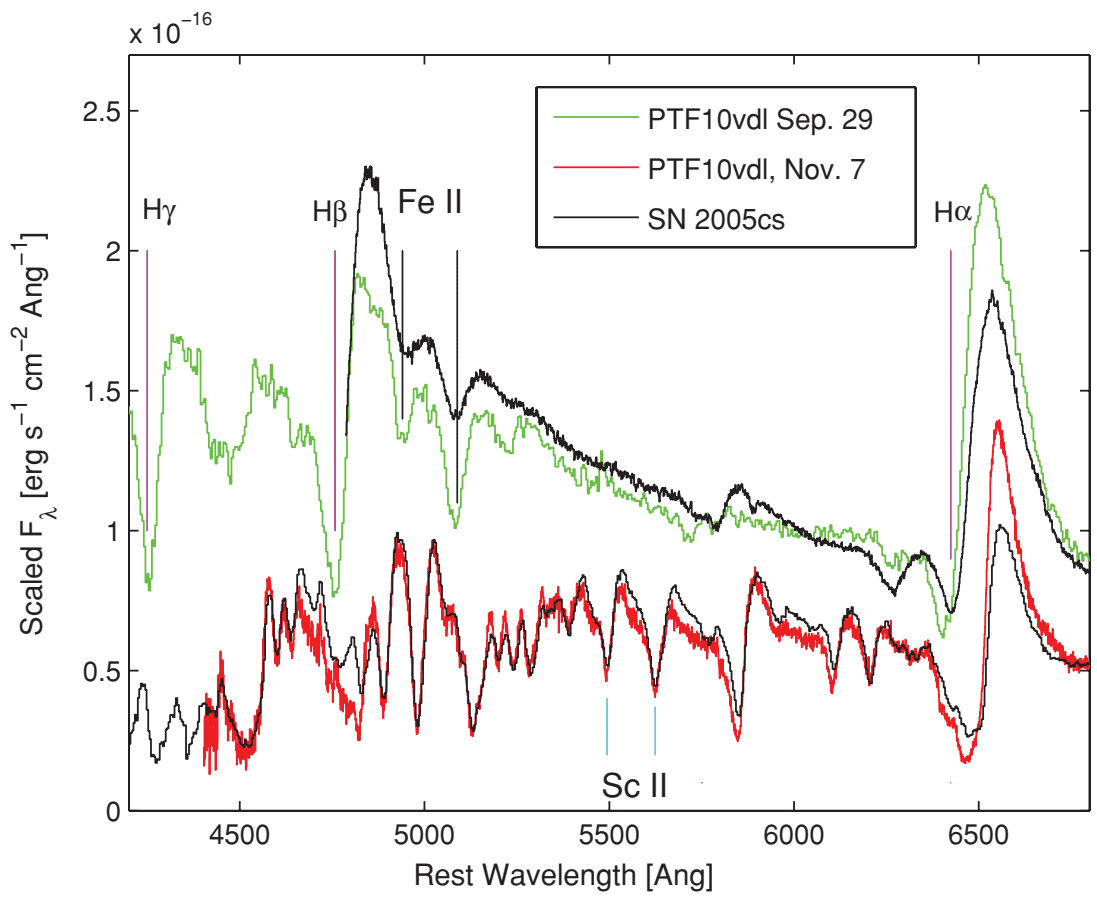

Figure 3. Spectral evolution of PTF10vdl. We show two later spectra of this event, covering the range $2-8$ weeks after explosion; a full description will be presented in a future publication. The first spectrum, obtained with the Low-Resolution Spectrograph mounted on the HET, is the first to show a typical spectrum for an SN II during the plateau phase (e.g., Filippenko 1997). Expansion velocities derived from minima of developing Fe lines are relatively low $\left(\sim 4700 \mathrm{~km} \mathrm{~s}^{-1}\right)$. As the object ages on the plateau, as seen in the second spectrum obtained with the DEIMOS spectrograph mounted on the Keck-II telescope, weaker lines of other elements develop; velocities measured from the Sc II lines are very low $\left(\sim 1800 \mathrm{~km} \mathrm{~s}^{-1}\right)$. The velocity evolution we measure is consistent with the trends seen in other low-luminosity SNe II-P (e.g., Pastorello et al. 2004, 2009); direct comparison is shown with spectra of SN 2005cs (black lines) obtained 6 days (top) and 33 days (bottom) after peak (from Pastorello et al. 2009).

(A color version of this figure is available in the online journal.) 


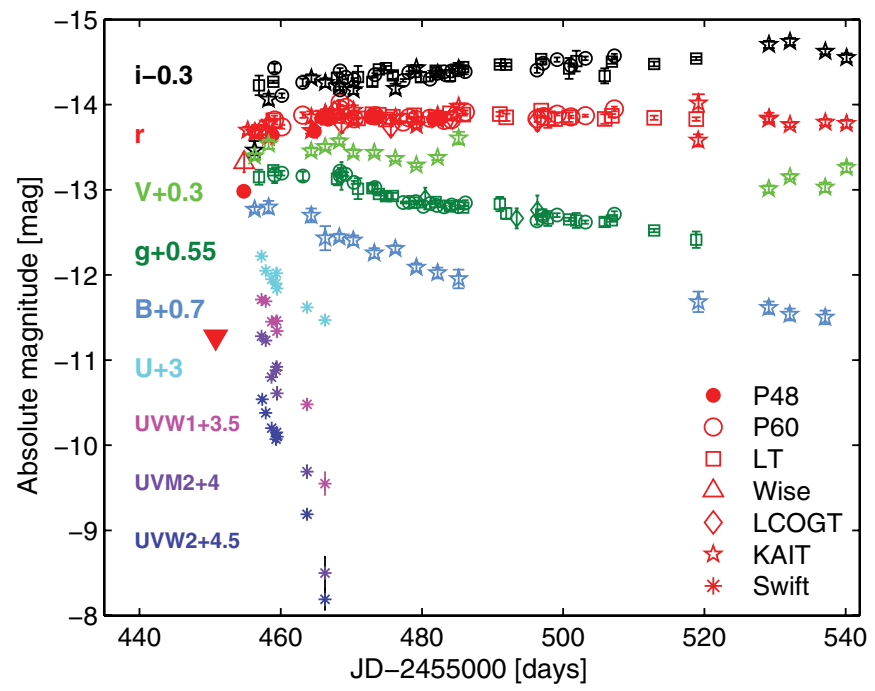

Figure 4. Light curve of PTF10vdl. P48 points obtained from the survey data using the "white glove" photometry pipeline (D. Poznanski et al. 2011, in preparation) daily averages are shown as red filled circles and the last nondetection as a downward-facing triangle. Ground-based BgVri and Swift $U, U V W 1, U V M 2$, and $U V W 2$ measurements are shown in blue, dark green, light green, red, black, cyan, magenta, purple, and indigo, respectively; the data source is indicated by the marked shape. KAIT I was transformed to the SDSS $i$-band grid to match $i$-band data from other sources; KAIT unfiltered and all $r / R$-band data were offset to align with the P60/LT data calibrated onto the SDSS $r$-band zeropoint. All data are reported on the absolute scale assuming a distance modulus of $\mu=32.86 \mathrm{mag}$ (from NED). For clarity, $i, V, g, B, U$, $U V W 1, U V M 2$, and $U V W 2$ data are offset by $-0.3,0.3,0.55,0.7,3,3.5,4$, and $4.5 \mathrm{mag}$, respectively. The rapid rise is followed by a constant plateau, the hallmark of SNe II-P. The absolute $r$-band plateau luminosity is remarkably faint, $M_{R}=-13.85 \mathrm{mag}$, comparable only to SN 1999br (Pastorello et al. 2004).

\section{RESULTS}

The combination of the PTF real-time analysis pipeline with rapid-response follow-up programs enables us to launch multiwavelength monitoring campaigns of optical transients, such as PTF10vdl, within hours of discovery. Since the observations of this particular object are still ongoing, a full discussion will be presented in future publications. Here, we report on the aspects that are unique to the rapid follow-up campaign we conducted.

Our data set includes a remarkable early-time spectrum (within 2 days of discovery; Figure 2), rarely obtained for $\mathrm{SNe}$ II in the past. It is similar to observations of a handful of other SNe II observed at comparably early ages. In particular, thermally excited He II lines are strongly detected, and they disappear within a few days after the explosion as the expanding photosphere cools. ${ }^{23}$ To the best of our knowledge, these are the earliest spectra ever obtained of an underluminous SN II-P. For example, spectroscopic coverage of SN 2005cs in M51, the best-observed member of this subclass, probably began about a day later than our earliest spectrum, and He II lines are not seen (Pastorello et al. 2009).

Our photometric coverage of this event extends from the far UV to the $i$ band, tracing the rise of the light curve onto the plateau with excellent temporal sampling. We measure an $r$-band plateau absolute magnitude of $M_{r}=-13.85$, which is remarkably faint; only a single other SN II-P, SN 1999br

\footnotetext{
23 We note that line identification in very early SN II spectra, and in particular, the relative contributions of $\mathrm{He}$ II, N II, and $\mathrm{O}$ II, is a matter of some debate (e.g., Quimby et al. 2007; Baron et al. 2007; Dessart et al. 2008); further analysis of our data in conjunction with detailed models is encouraged.
}

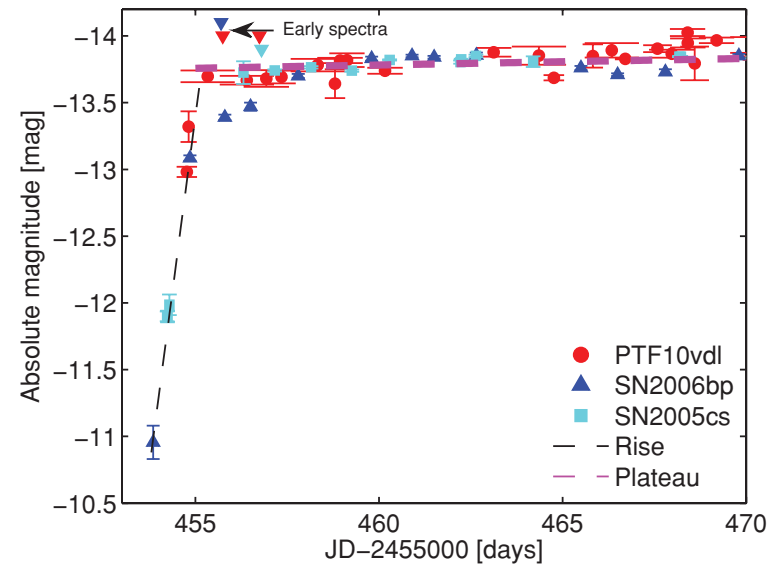

Figure 5. Early-time light curves of SNe II-P. We compare our $R$-band light curve of PTF10vdl (red) with those of the normal SN II-P 2006bp (daily averages from Quimby et al. 2007; blue) and the underluminous SN 2005cs (Pastorello et al. 2009; cyan). We have scaled the flux to align the plateau luminosities (magenta dashed line). As shown by Pastorello et al. (2009), the rapid rise phase of SNe 2005cs and 2006bp is consistent with a single linear slope (in magnitude vs. time; black dashed line), and we have adjusted the estimated explosion date of PTF10vdl (which is not as well constrained as those of the other events) so that the earliest observations tracing the rise fall on the same slope. It appears as if the underluminous events, PTF10vdl and SN 2005cs, transition sharply from a rise to a plateau, compared to the more luminous SN 2006bp. Additional events (and even better data) are required to determine if this is a general trend. The dates of the earliest spectra for these three events (from Pastorello et al. 2006; Quimby et al. 2007, and this work) are marked with triangles having colors matching the figure legend.

(Pastorello et al. 2004), has a comparable plateau luminosity $\left(M_{V}=-13.76 \mathrm{mag}\right)$. Linear decline rates measured in the $U V W 2, U V M 2, U V W 1, U, B, g, V, r$, and $i$ bands are 0.2509 , $0.2995,0.2375,0.0743,0.0191,0.0149,0.0120,-0.0008$, and -0.0043 mag day $^{-1}$, respectively. Bands bluer than $g$ show a rapid decline starting with our earliest data points, while the $i$ band exhibits a slow rise during the initial phase of the plateau.

The only two other SNe II-P observed this early with welldefined plateau light curves (cf. the peculiar SN 1987A) were SN 2005cs (underluminous; Pastorello et al. 2009) and SN 2006bp (normal; Quimby et al. 2007). Comparison with PTF10vdl shows a possible trend: it seems that both underluminous events (SN 2005cs and PTF10vdl) have a fast rise with a sharp onset of the flat plateau, while the more luminous SN 2006bp has a more gradual transition, with the fast rise leveling onto the plateau over a span of a few days (Figure 5; see also Figure 3 of Pastorello et al. 2009). Future early (and dense) monitoring of additional SNe II-P should clarify whether there is a correlation between the plateau luminosity and the morphology of the earlytime light curve; presumably such differences, if they exist, reflect the physical structure of the outermost layers of the exploding star. At these early times, line emission is weak and the $r$-band flux is dominated by continuum emission on the Rayleigh-Jeans tail of the Planck curve, so its evolution should mirror that of the bolometric luminosity.

Type II-P SNe have been shown to be promising "standard candles" using an empirical relation between the photospheric velocity (measured from weak absorption lines, e.g., of Fe II), a color term (that traces dust extinction and intrinsic variance), and the luminosity (Hamuy \& Pinto 2002; Nugent et al. 2006; Poznanski et al. 2009, 2010). While low-luminosity events such as PTF10vdl are expected to be rare in flux-limited cosmological SN samples, it is instructive to examine whether this event follows the general relation. Using the parameters 
from Poznanski et al. (2009), we find that PTF10vdl is a significant outlier, $\sim 1$ mag away from the benchmark set by higher luminosity SNe II-P. Its velocity on the plateau (1800 $\mathrm{km} \mathrm{s}^{-1}$; Figure 5) translates into a positive luminosity correction of $\sim 2 \mathrm{mag}$, with an additional $0.25 \mathrm{mag}$ from its color correction. However, its plateau luminosity is more than 3 mag fainter than that of the average SN II-P. We note that SN 2005cs (which is somewhat brighter than PTF10vdl, but still considered among the low-luminosity SN II-P group; Pastorello et al. 2009) does follow the cosmological relation. Even if some fainter $\mathrm{SNe}$ II-P like PTF10vdl violate the correlations established at higher luminosities, this is unlikely to impede future efforts to use $\mathrm{SNe}$ II-P as cosmological beacons, as such faint objects are rare in flux-limited samples. On the other hand, this apparent anomaly motivates further studies of low-luminosity SNe II-P and continued investigation of the physics driving observed correlations (e.g., Kasen \& Woosley 2009). The combination of early spectroscopic observations and UV/optical light curves makes PTF10vdl an attractive target for such studies, as well as for comparison with detailed explosion models (e.g., Baron et al. 2004; Dessart et al. 2008).

Our Swift observations resulted in solid detections of PTF10vdl in all UV filters (Kasliwal et al. 2010a) and a nondetection in X-rays. CARMA and EVLA observations also resulted in upper limits (Carpenter et al. 2010; Kasliwal et al. 2010a). It is interesting to note that both Swift and EVLA observations were delayed by $\sim 48 \mathrm{hr}$ due to technical limitations that should generally not apply; thus, future PTF events should be observed even sooner, increasing the exciting prospect of X-ray, $\mathrm{UV}$, and radio detection of prompt, nonthermal radiation from cosmic explosions detected by PTF.

Note that the LOSS (Filippenko et al. 2001) discovered the same object $\sim 1$ day after our PTF discovery (Lin et al. 2010), but CBET procedures prevented the dissemination of this information for several days, until it was announced as SN 2010id. It thus seems that high-cadence observations of nearby galaxies by LOSS should also be able to contribute to future discoveries at very early times.

\section{DISCUSSION AND CONCLUSIONS}

Early detection and rapid follow-up observations, as demonstrated with PTF discoveries, are interesting for a number of reasons. The first electromagnetic manifestation of the explosions of massive stars as core-collapse $\mathrm{SNe}$ would be the process of the outgoing shock emerging from the stellar envelope (so-called shock-breakout), followed by a period of rapid radiative cooling. PTF optical data alone can now be used to detect such explosions during the first hours after they occur and to trigger space-based follow-up observations in the UV and X-rays. At least for exploding red supergiants, the fewhour timescale should allow observations of the tail (at least) of the shock-breakout process as well as the subsequent cooling, enabling measurement of the radius of the exploding star and determination of its atmospheric composition (e.g., Rabinak \& Waxman 2011), and perhaps even constraining shock-breakout physics (e.g., Katz et al. 2010; Nakar \& Sari 2010). Such data would be extremely valuable to guide and constrain massivestar evolution models. It is not yet clear whether the observations of PTF10vdl presented here would prove useful in this respect. Very early observations of thermonuclear SNe Ia (for recent PTF examples, see Cooke et al. 2011; Howell et al. 2010; Kasliwal et al. 2010c) would provide constraints on the progen- itors of these important cosmic distance estimators (e.g., Kasen 2010).

Several more exotic scenarios predict fast transients on $\sim 1$ day timescales (e.g., compact-object mergers, Metzger et al. 2010; accretion-induced collapse of a white dwarf to a neutron star, Metzger et al. 2009). If these transients emit enough red light to be detected by the PTF $r$-band survey, rapid followup observations would be critical for studying their properties during their fleetingly short luminous phases.

An important aspect common to the processes mentioned above (SNe, compact-object mergers) is that they are expected to be leading sources of gravitational waves in the advanced LIGO/VIRGO bands, as well as potential sources of cosmic neutrinos. A narrow timing window based on coincidence with PTF optical detections would allow searching for a signal over a significantly shorter stream of background noise, thereby reducing the minimal detection threshold and increasing the effective sensitivity of existing detectors (e.g., Kochanek \& Piran 1993; Cowen et al. 2010). While PTF will no longer be active during the next LIGO science run expected to begin in 2014, developing and polishing the real-time capability will be highly beneficial for synergy between LIGO and future successors of PTF, as well as between PTF and current neutrino experiments such as Ice Cube.

To summarize, we have described the PTF operational setup for real-time object detection and rapid multiwavelength followup observations. As an example, we have presented the realtime discovery of the optical transient PTF10vdl by the PTF survey, and results from a rapid-response multiwavelength follow-up campaign. Our observations cover the very early phase (first days) following the explosion of an underluminous Type II core-collapse SN, probably discovered within $24 \mathrm{hr}$ of explosion, including very early spectra. Our results demonstrate that the PTF wide-field optical survey, in synergy with ToO programs using space- and ground-based facilities, now enables us to conduct rapid multiwavelength investigations of optical transients in a manner previously carried out only for highenergy transients (GRBs). Our observations indicate that we should expect observational breakthroughs in the study of $\mathrm{SNe}$ and other optical transients similar to those recently achieved for GRBs.

This paper is dedicated to the memory of our dear colleague, J. Jacobsen, who contributed significantly to the success of this project.

The Palomar Transient Factory project is a scientific collaboration between the California Institute of Technology, Columbia University, Las Cumbres Observatory, the Lawrence Berkeley National Laboratory, the National Energy Research Scientific Computing Center, the University of Oxford, and the Weizmann Institute of Science. Weizmann Institute participation in PTF is supported in part by grants from the Israeli Science Foundation (ISF) to A.G.-Y. Joint Weizmann-Caltech activity is supported by a grant from the Binational Science Foundation to A.G.-Y. and S.R.K. Support for Weizmann-UK collaborative work is provided by a Weizmann-UK "making connections" grant to A.G.-Y. and M.S. Joint activity by A.G.-Y. and P.A.M. is supported by a Weizmann-MINERVA grant. A.G.-Y. further acknowledges support from an EU/FP7 Marie Curie IRG Fellowship. E.O.O. and D.P. are grateful to NASA for Einstein Fellowships. The work of A.V.F.'s group at UC Berkeley is funded by US National Science Foundation (NSF) grant 
AST-0908886, the TABASGO Foundation, Gary and Cynthia Bengier, and the Richard and Rhoda Goldman Fund.

LAIWO, a wide-angle camera operating on the $1 \mathrm{~m}$ telescope at the Wise Observatory, Israel, was built at the Max Planck Institute for Astronomy (MPIA) in Heidelberg, Germany, with financial support from the MPIA, grants from the German Israeli Science Foundation for Research and Development, and the ISF. KAIT and its ongoing operation were made possible by donations from Sun Microsystems, Inc., the Hewlett-Packard Company, AutoScope Corporation, Lick Observatory, the NSF, the University of California, the Sylvia \& Jim Katzman Foundation, and the TABASGO Foundation. The National Energy Research Scientific Computing Center, which is supported by the Office of Science of the U.S. Department of Energy under Contract No. DE-AC02-05CH11231, provided staff, computational resources, and data storage for this project. P.E.N. acknowledges support from the U.S. Department of Energy Scientific Discovery through Advanced Computing program under contract DE-FG02-06ER06-04.

Some of the data presented herein were obtained at the W. M. Keck Observatory, which is operated as a scientific partnership among the California Institute of Technology, the University of California, and NASA; the observatory was made possible by the generous financial support of the W. M. Keck Foundation. We thank the staffs of the many observatories at which data were obtained for their excellent assistance. This research has made use of the NASA/IPAC Extragalactic Database (NED), which is operated by the Jet Propulsion Laboratory, California Institute of Technology, under contract with NASA.

\section{REFERENCES}

Baron, E., Branch, D., \& Hauschildt, P. H. 2007, ApJ, 662, 1148

Baron, E., Nugent, P. E., Branch, D., \& Hauschildt, P. H. 2004, ApJ, 616, L91 Carpenter, J. M. 2010, ATel, 2473, 1

Carpenter, J. M., et al. 2010, ATel, 2863

Cenko, S. B., et al. 2006, PASP, 118, 1396

Cenko, S. B., et al. 2011, ApJ, submitted (arXiv:1103.0779)

Cooke, J., et al. 2011, ApJ, 727, L35
Corsi, A., et al. 2011, ApJ, submitted (arXiv:1101.4208)

Cowen, D. F., Franckowiak, A., \& Kowalski, M. 2010, Astropart. Phys., 33, 19 Dessart, L., et al. 2008, ApJ, 675, 644

Filippenko, A. V. 1982, PASP, 94, 715

Filippenko, A. V. 1997, ARA\&A, 35, 309

Filippenko, A. V., Li, W. D., Treffers, R. R., \& Modjaz, M. 2001, in ASP Conf. Ser. 246, Small Telescope Astronomy on Global Scales, ed. B. Paczynski, W.-P. Chen, \& C. Lemme (San Francisco, CA: ASP), 121

Ganeshalingam, M., et al. 2010, ApJS, 190, 418

Hamuy, M., \& Pinto, P. A. 2002, ApJ, 566, L63

Hook, I. M., Jørgensen, I., Allington-Smith, J. R., Davies, R. L., Metcalfe, N., Murowinski, R. G., \& Crampton, D. 2004, PASP, 116, 425

Howell, D. A., et al. 2010, ATel, 2934, 1

Kasen, D. 2010, ApJ, 708, 1025

Kasen, D., \& Woosley, S. E. 2009, ApJ, 703, 2205

Kasliwal, M. M., et al. 2010a, ATel, 2862

Kasliwal, M. M., et al. 2010b, ATel, 2864

Kasliwal, M. M., et al. 2010c, ATel, 2957

Kasliwal, M. M., et al. 2011, ApJ, 730, 134

Katz, B., Budnik, R., \& Waxman, E. 2010, ApJ, 716, 781

Kochanek, C. S., \& Piran, T. 1993, ApJ, 417, L17

Law, N. M., et al. 2009, PASP, 121, 1395

Lin, K., et al. 2010, CBET, 2467

Metzger, B. D., Piro, A. L., \& Quataert, E. 2009, MNRAS, 396, 1659

Metzger, B. D., et al. 2010, MNRAS, 406, 2650

Nakar, E. 2007, Phys. Rep., 442, 166

Nakar, E., \& Sari, R. 2010, ApJ, 725, 904

Nugent, P., et al. 2006, ApJ, 645, 841

Ofek, E. O., et al. 2010, ApJ, 724, 1396

Pastorello, A., et al. 2004, MNRAS, 347, 74

Pastorello, A., et al. 2006, MNRAS, 370, 1752

Pastorello, A., et al. 2009, MNRAS, 394, 2266

Piran, T. 2004, Rev. Mod. Phys., 76, 1143

Poole, T. S., et al. 2008, MNRAS, 383, 627

Poznanski, D., Nugent, P. E., \& Filippenko, A. V. 2010, ApJ, 721, 956

Poznanski, D., et al. 2009, ApJ, 694, 1067

Quimby, R. M., Wheeler, J. C., Höflich, P., Akerlof, C. W., Brown, P. J., \& Rykoff, E. S. 2007, ApJ, 666, 1093

Rabinak, I., \& Waxman, E. 2011, ApJ, 728, 63

Rau, A., et al. 2009, PASP, 121, 1334

Smith, A. M., et al. 2011, MNRAS, 412, 1309

Soderberg, A. M., et al. 2008, Nature, 453, 469

Springob, C. M., Haynes, M. P., Giovanelli, R., \& Kent, B. R. 2005, ApJS, 160, 149

Stritzinger, M., et al. 2002, AJ, 124, 2100

Woosley, S. E., \& Bloom, J. S. 2006, ARA\&A, 44, 507 